\title{
Singular open book structures from real mappings
}

Raimundo N. Araújo dos Santos ${ }^{1 *}$, Ying Chen ${ }^{2 \dagger}$, Mihai Tibăr ${ }^{2 \neq}$

1 ICMC, Universidade de São Paulo, Av. Trabalhador São-Carlense 400, CP Box 668, 13560-970 São Carlos, São Paulo, Brazil

2 Mathématiques, UMR-CNRS 8524, Université Lille 1, 59655 Villeneuve d'Ascq, France

Received 8 February 2012; accepted 3 July 2012

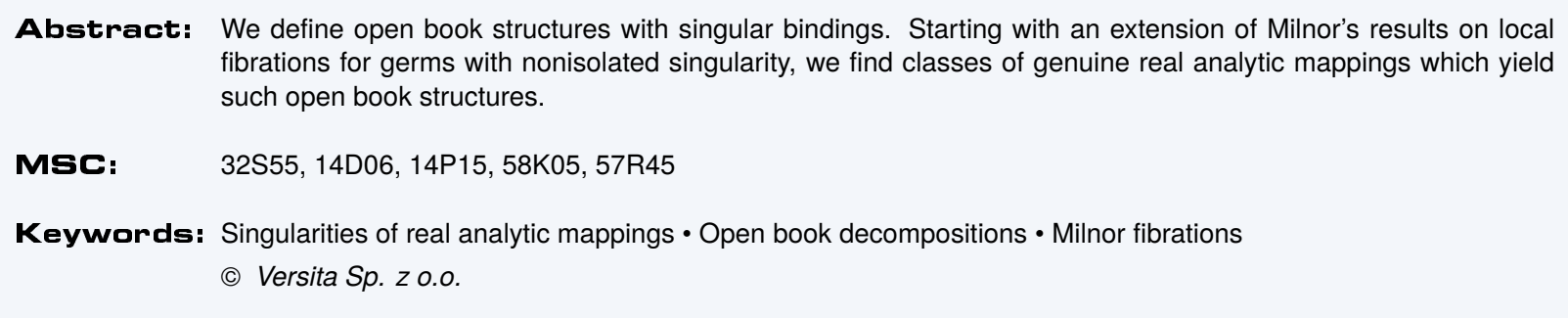

\section{Introduction and main results}

Let $\psi:\left(\mathbb{R}^{m}, 0\right) \rightarrow\left(\mathbb{R}^{p}, 0\right)$ be some analytic mapping germ, $m>p \geq 2$, and $V=\psi^{-1}(0)$. The isolated singularity case, i.e. Sing $\psi=\{0\}$, has been considered by Milnor; his result [14, Theorem 11.2] says that there exists a mapping

$$
S_{\varepsilon}^{m-1} \backslash K_{\varepsilon} \rightarrow S_{\delta}^{p-1}
$$

which is a locally trivial fibration and its diffeomorphism type is independent of the radius $\varepsilon>0$ (small enough) and of $0<\delta \ll \varepsilon$, where $K_{\varepsilon}=V \cap S_{\varepsilon}^{m-1}$. Moreover, the sphere $S_{\varepsilon}^{m-1}$ is endowed with a so-called higher open book structure with binding $K_{\varepsilon}$, see [1, Definition 2.1]. This is due to the trivial fibration structure induced by $\psi$ in some small neighbourhood $N$ of $K_{\varepsilon}$. The condition $V \cap \operatorname{Sing} \psi \subset\{0\}[1]$ is the most general one under which higher open

*E-mail: rnonato@icmc.usp.br

† E-mail: Ying.Chen@math.univ-lille1.fr

F E-mail: tibar@math.univ-lille1.fr 
book structures with smooth binding $K_{\varepsilon}$ exist. Classical open books, i.e. those for which the binding is smooth and has codimension 2, are used in the literature under various other names like fibered links, Neuwirth-Stallings pairs [12] or Lefschetz pencils, see e.g. [24].

In this paper we investigate the case when $\psi^{-1}(0)$ contains nonisolated singularities, thus the link $K_{\varepsilon}$ is no longer a manifold. Several recent papers considered this situation with respect to the existence of the Milnor fibration (1), see e.g. $[2,4,18]$. Let us first introduce the notion of singular open book structures.

\section{Definition 1.1.}

We say that the pair $(K, \theta)$ is a higher open book structure with singular binding on an analytic manifold $M$ of dimension $m-1 \geq p \geq 2$ if $K \subset M$ is a singular real subvariety of codimension $p$ and $\theta: M \backslash K \rightarrow S_{1}^{p-1}$ is a locally trivial smooth fibration such that $K$ admits a neighbourhood $N$ for which the restriction $\theta \uparrow_{M K}$ is the composition $N \backslash K \stackrel{h}{\rightarrow} B^{p} \backslash\{0\} \stackrel{s /\|s\|}{\longrightarrow} S_{1}^{p-1}$, where $h$ is a locally trivial fibration. The singular fibered link $K$ is called the binding and the (closures of) fibers of $\theta$ are called pages of the open book.

From the above definition it follows that $\theta$ is surjective. In the classical case of open books one has $p=2, K \subset M$ is a 2-codimensional submanifold which admits a neighbourhood $N$ diffeomorphic to $B^{2} \times K$ for which $K$ is identified with $\{0\} \times K$ and the restriction $\theta \Gamma_{N \backslash K}$ is the following composition with the natural projections:

$$
N \backslash K \stackrel{\text { diffeo }}{\simeq}\left(B^{2} \backslash\{0\}\right) \times K \stackrel{\text { proj }}{\longrightarrow} B^{2} \backslash\{0\} \longrightarrow S^{1} .
$$

Our new definition of open book with singular binding preserves the two main aspects of the classical definitions:

(a) The complement of the link fibers over the unit sphere, with codimension $p-1$ fibres.

(b) This fibration has a regularity property in the neighbourhood of the link, namely, it is induced by a more refined fibration with codimension $p$ fibres.

These two properties turn out to be equally important and independent whenever $V$ contains nonisolated singularities. Our proofs will therefore contain two parts, corresponding to demonstration of properties (a) and (b).

Whenever $\psi$ is the pair $(\operatorname{Re} f, \operatorname{Im} f)$ associated to a holomorphic function germ $f:\left(\mathbb{C}^{n}, 0\right) \rightarrow(\mathbb{C}, 0)$, one has Sing $\psi \subset$ $V=f^{-1}(0)$ and that the natural mapping

$$
\frac{f}{|f|}: S_{\varepsilon}^{2 n-1} \backslash K_{\varepsilon} \rightarrow S_{1}^{1}
$$

is a $C^{\infty}$ locally trivial fibration $[14, \S 4]$. If $V$ contains nonisolated singularities, to have an open book decomposition we also need property (b). In [7], Hamm and Lê proved a nontrivial fact that holomorphic functions have the Thom $\left(\mathrm{a}_{f}\right)$ property along a certain stratification of $V$ what guarantees property (b).

If we consider a holomorphic mapping $\mathbb{C}^{n} \rightarrow \mathbb{C}^{p}$ with $p>1$ then there might not exist fibrations like $(2)^{1}$ or the Thom regularity may fail ${ }^{2}$ along $V$. In the purely real analytic setting, the mapping $\psi$ may have neither property (a) nor property (b). In Section 2 we formulate an extension of [14, Theorem 11.2] to the open books with singular binding from Definition 1.1, the proof of which is derived from careful revisiting of Milnor's construction. This is further used as a basis for our main results. In order to state them, we need the following definitions.

\section{Definition 1.2.}

Let $U \subset \mathbb{R}^{m}$ be an open set and $\rho: U \rightarrow \mathbb{R}_{\geq 0}$ a proper analytic function. We say that the set of $\rho$-nonregular points ${ }^{3}$ of an analytic mapping $\Psi: U \rightarrow \mathbb{R}^{p}$ is the set of non-transversality between $\rho$ and $\Psi$, i.e. $M(\Psi)=\left\{x \in U: \rho \not \mathbb{x}_{x} \Psi\right\}$. Similarly, the set of $\rho$-nonregular points of the mapping $\Psi /\|\Psi\|: U \backslash V \rightarrow S_{1}^{p-1}$ is the set $M(\Psi /\|\Psi\|)=\operatorname{closure}\{x \in$ $\left.U \backslash V: \rho \oiint_{x} \Psi /\|\Psi\|\right\}$.

1 In case of an ICIS one does not have the fibration (2).

2 E.g. like shown by an example in [7].

3 Sometimes called the Milnor set of $\Psi$, e.g. in [15]; the name $\rho$-regularity has been introduced in [21]. 
Let us remind that, by definition, two mappings on an open $U \subset \mathbb{R}^{m}$ are called transversal at some point $x \in U$ if they are both non-singular at $x$ and their well-defined tangent spaces at $x$ are transversal in $\mathbb{R}^{m}$. In particular, the singular locus of each of the mappings is included in their non-transversality locus.

The $\rho$-regularity from Definition 1.2 is a basic tool, used by many authors (Thom, Milnor, Mather, Looijenga, Bekka, etc.) in the local stratified setting as well as at infinity, see e.g. $[15,21-23]$ in order to produce locally trivial fibrations. See also [4]. Thom has called such $\rho$ the fonction tapissante. As in [2], in this paper we shall use as a function $\rho$ the Euclidean distance function and its open balls and spheres of radius $\varepsilon$, denoted by $B_{\varepsilon}$ and $S_{\varepsilon}$ respectively. The open set $U$ from Definition 1.2 will be the small ball $B_{\varepsilon}$, unless otherwise stated.

\section{Theorem 1.3.}

Let $\psi:\left(\mathbb{R}^{m}, 0\right) \rightarrow\left(\mathbb{R}^{p}, 0\right)$ be an analytic mapping germ, $m>p \geq 2$, such that $\operatorname{codim} V=p$. Suppose that

$$
\overline{M(\psi) \backslash V} \cap V=\{0\} .
$$

If $M(\psi /\|\psi\|)=\emptyset$ then $\left(K_{\varepsilon}, \psi /\|\psi\|\right)$ is an open book structure with singular binding on $S_{\varepsilon}^{m-1}$, independent (up to isotopies) of $\varepsilon>0$ small enough.

Condition (3) allows nonisolated singularities, more precisely, it implies $M(\psi)=A \cup B$ where $A \subset V$ and $B \cap V \subset\{0\}$, and both $A$ and $B$ may be of positive dimension; see Figure 1. We also have Sing $\psi \subset M(\psi)$. Therefore Theorem 1.3 represents a simultaneous extension of [1, Theorem 2.2], where the singular locus was of type B, and of [2, Proposition 5.3] or $[4 \text {, Theorem 5.3 }]^{4}$, where the singular locus was of type A. We discuss in Section 3 other particular cases and the relations to [18].

It turns out that condition ${ }^{5}$ (3) insures the existence of the locally trivial fibration $N \backslash K \stackrel{h}{\rightarrow} B^{p} \backslash\{0\}$ from Definition 1.1 and is implied by the Thom regularity at $V$. We shall discuss this relation and other criteria in Section 5 . We show in Example 5.1 how to check this condition directly.

In order to produce a new class of higher dimensional purely real examples, we use the theory of mixed functions, the real analytic mappings $\mathbb{R}^{2 n} \simeq \mathbb{C}^{n} \rightarrow \mathbb{C} \simeq \mathbb{R}^{2}$, recently developed by Mutsuo Oka (see [16,17] and our footnote at Section 4). The necessary definitions are given in Section 4. We only point out here that for mixed functions, unlike the holomorphic ones, the notion of polar weighted-homogeneous is different and independent from radial weighted-homogeneous. The later is discussed in Section 3 and is one of the hypotheses of Corollary 3.3. The former occurs in the following statement (discussed and proved in Section 4).

\section{Theorem 1.4.}

Let $f: \mathbb{C}^{n} \rightarrow \mathbb{C}$ be a non-constant mixed polynomial which is polar weighted-homogeneous, $n \geq 2$, such that $\operatorname{codim}_{\mathbb{R}} V=2$. Then $\left(K_{\varepsilon}, f /\|f\|\right)$ is an open book structure with singular binding on $S_{\varepsilon}^{2 n-1}$, independent (up to isotopies) of $\varepsilon>0$ small enough.

We finally present some other new classes of examples in Section 5, one of them by using a Thom-Sebastiani type statement, Proposition 5.2, which represents a new result in the real context.

\section{Note 1.5.}

One may work in a slightly more general setting than Definition 1.1, as follows. Instead of a manifold, $M$ may be a connected compact real analytic set with Sing $M \subset K$. In this paper $M$ is the link of $\left(\mathbb{R}^{m}, 0\right)$, hence a sphere, but we may also consider a real analytic germ $(X, 0) \subset\left(\mathbb{R}^{m}, 0\right)$ with connected link $M$ with respect to some distance function

4 Compare the result [2, Proposition 5.3 and Remark 5.4] to [4, Theorem 5.3(1), (2) and Remark 5.7]. Then compare the comments [4, p. 423, lines 5-8] to the actual content of [2, Section 5].

5 Massey [13] used condition (3) in conjunction with Sing $\psi \subset V$ in order to get a full tube fibration (5). 
which is not necessarily the Euclidean one, and mappings $\psi:(X, 0) \rightarrow\left(\mathbb{R}^{p}, 0\right)$ such that Sing $X \subset \psi^{-1}(0)$. Then one has to modify Milnor's proof in subsection 2.1.2 so that the vector field along which one blows the tube to the sphere is tangent to $X$. See also Remark 2.2.

Even more generally, if one considers any singular stratified space $X$ and its link $M$, then Milnor's method reviewed in subsection 2.1 still works, i.e. can be extended (by using classical technical devices of radial vector fields) to stratified transversality and stratified vector fields. This yields what one could call open book structures with singular pages.

\section{Milnor's method and open book structures}

In the real analytic setting, Milnor observed that the fibration (1) need not be induced by the mapping $\theta=\psi /\|\psi\|$. He gave an example [14, p. 99] of $\psi$ with isolated singularity, $m=p=2$ and $K_{\varepsilon}=\emptyset$, showing that the mapping

$$
\frac{\psi}{\|\psi\|}: S_{\varepsilon}^{m-1} \backslash K_{\varepsilon} \rightarrow S_{1}^{1}
$$

is not a submersion, hence not a locally trivial fibration.

In the case of isolated singularity and $p=2$, several authors obtained sufficient conditions under which (4) is a fibration $[9,10,19,20]$ and provided with examples showing that the class of real mapping germs $\psi$ with isolated singularity satisfying them enlarges the class of holomorphic functions $f$. For a more general setting, $\operatorname{Sing} \psi \cap V \subset\{0\}$ and any $m \geq p \geq 2$, an existence criterion was given in [1] and shown to be more general than the previous ones from the literature.

From Definition 1.2 it follows that $M(\psi)$ is a relatively closed analytic set containing the singular set Sing $\psi$. As for $M(\psi /\|\psi\|)$, it is by definition closed but does not necessarily include Sing $\psi$. We nevertheless have $M(\psi /\|\psi\|) \backslash V \subset$ $M(\psi) \backslash V$, since $\rho \pitchfork_{x} \psi$ implies $\rho \pitchfork_{x} \psi /\|\psi\|$ for $x \notin V$. In the following we tacitly conceive these non-regularity sets as set germs at the origin.

\section{Theorem 2.1 (after [14]).}

Let $\psi:\left(\mathbb{R}^{m}, 0\right) \rightarrow\left(\mathbb{R}^{p}, 0\right), m>p \geq 2$, be an analytic mapping germ with Sing $\psi \subset V$, $\operatorname{codim}_{\mathbb{R}} V=p$ and satisfying condition (3). Then there exists a higher open book structure with singular binding $\left(K_{\varepsilon}, \theta\right)$ on $S_{\varepsilon}^{m-1}$, which is independent of $\varepsilon>0$ small enough, up to isotopies.

\subsection{Revisiting Milnor's method}

In the case of mapping germs $\psi:\left(\mathbb{R}^{m}, 0\right) \rightarrow\left(\mathbb{R}^{p}, 0\right)$, in Milnor's proof [14, pp.97-99, Theorem 11.2] of the existence of a locally trivial fibration (1), one may distinguish two key parts, already pointed out in [2, Section 5]. We shortly review them below in order to show how they apply to the more general situation displayed in Theorem 2.1.

\subsubsection{Existence of the tube fibration}

Assume that there exists $\varepsilon_{0}>0$ such that the mapping

$$
\psi \uparrow: \bar{B}_{\varepsilon}^{m} \cap \psi^{-1}\left(\bar{B}_{\eta}^{p} \backslash\{0\}\right) \rightarrow \bar{B}_{\eta}^{p} \backslash\{0\}
$$

is a surjective locally trivial $C^{\infty}$-fibration, for all $0<\varepsilon \leq \varepsilon_{0}$ and $0<\eta=\eta(\varepsilon) \ll \varepsilon$. We shall call it the tube fibration. In the case Sing $\psi=\{0\}$ considered by Milnor, $V$ is transverse to all small enough spheres and therefore any such sphere is also transverse to all nearby fibres, which are moreover non-singular. By Ehresmann's theorem for manifolds with boundary, one concludes the existence of the tube fibration. 


\subsubsection{Existence of a fibration in the exterior of the tube}

This is Milnor's proof of "inflating" the empty tube $\bar{B}_{\varepsilon}^{m} \cap \psi^{-1}\left(S_{\eta}^{p-1}\right)$ to the sphere $S_{\varepsilon}^{m-1} \backslash K_{\varepsilon}$. Milnor explains in [14, p. 99] that, given a real analytic mapping $\psi$, one may construct, like in [14, Lemmas 11.3, 5.9, 5.10], a nowhere zero $C^{\infty}$-vector field $v(x)$ on $\bar{B}_{\varepsilon}^{m} \backslash \psi^{-1}\left(B_{\eta}^{p}\right)$ satisfying the following two conditions: $\langle x, v(x)\rangle>0$ and $\left\langle\operatorname{grad}\|\psi(x)\|^{2}, v(x)\right\rangle>0$. The first condition says that $v(x)$ is transverse to all small spheres and points outwards, and the second condition says that the mapping $\|\psi(x)\|^{2}$ increases along the flow. This vector field may be integrated and produces a diffeomorphism

$$
\gamma: \bar{B}_{\varepsilon}^{m} \cap \psi^{-1}\left(S_{\eta}^{p-1}\right) \rightarrow S_{\varepsilon}^{m-1} \backslash \psi^{-1}\left(B_{\eta}^{p}\right)
$$

This procedure combines the position vector field $x$ with the gradient vector field grad $\|\psi\|^{2}$ and works if the latter is nowhere zero in the neighbourhood of $\psi^{-1}\left(B_{\eta}^{p}\right)$, if the two vector fields never point in the opposite directions [14, Corollary 3.4], and the empty tube $\bar{B}_{\varepsilon}^{m} \cap \psi^{-1}\left(S_{\eta}^{p-1}\right)$ is a manifold with boundary. Milnor uses this construction for holomorphic functions $f$ where Sing $f \subset V$ and for real mappings with $\operatorname{Sing} \psi=\{0\}$. Milnor's technique holds locally at 0 in the more general setting Sing $\psi \subset V$.

\subsubsection{Conclusions}

If the tube fibration (5) exists, then its restriction to the empty tube

$$
\psi \uparrow: \bar{B}_{\varepsilon}^{m} \cap \psi^{-1}\left(S_{\eta}^{p-1}\right) \rightarrow S_{\eta}^{p-1}
$$

is a locally trivial $C^{\infty}$-fibration too. If the inflating procedure works, then the diffeomorphism (6) induces a mapping $\mu: S_{\varepsilon}^{m-1} \backslash \psi^{-1}\left(B_{\eta}^{p}\right) \rightarrow S_{\eta}^{p-1}$ which is a locally trivial fibration and coincides with $\psi$ on $S_{\varepsilon}^{m-1} \cap \psi^{-1}\left(S_{\eta}^{p-1}\right)$. This may be composed with the mapping $s /\|s\|: S_{\eta}^{p-1} \rightarrow S_{1}^{p-1}$ and yields a locally trivial fibration

$$
\mu^{\prime}: S_{\varepsilon}^{m-1} \backslash \psi^{-1}\left(B_{\eta}^{p}\right) \rightarrow S_{1}^{p-1}
$$

\subsection{Proof of Theorem 2.1}

The hypothesis $\overline{M(\psi) \backslash V} \cap V=\{0\}$ is equivalent to the existence of a neighbourhood $\mathcal{N}$ of $V \backslash\{0\}$ such that $M(\psi) \cap \mathcal{N} \backslash V=\emptyset$ (see Figure 1). Together with Sing $\psi \subset V$, these imply that for any $\varepsilon>0$ small enough, there exists some positive $\eta \ll \varepsilon$ such that the mapping (5) is a proper submersion over the pointed open ball $B^{p} \backslash\{0\}$. Indeed, the properness follows since the restriction of $\psi$ to $\bar{B}_{\epsilon}^{m} \backslash V$ is proper, and "submersion" is a consequence of the condition Sing $\psi \subset V$ and transversality of the fibres to the boundary $S_{\varepsilon}^{m-1}=\partial \bar{B}_{\epsilon}^{m}$. It then follows that this restriction is also surjective. Just like Milnor did, we may now apply Ehresmann's theorem $[5,25]$ for manifolds with boundary to conclude the existence of the locally trivial fibration (5). ${ }^{6}$

To show now that the fibration (8) extends to an open book structure on $S_{\varepsilon}^{m-1}$ one must produce the map $\theta$, cf. Definition 1.1. The fibration (8) may be glued along $S_{\varepsilon}^{m-1} \cap \psi^{-1}\left(S_{\eta}^{p-1}\right)$ to the locally trivial fibration $S_{\varepsilon}^{m-1} \cap \psi^{-1}\left(\bar{B}_{\eta}^{p} \backslash\{0\}\right) \rightarrow$ $\bar{B}_{\eta}^{p} \backslash\{0\}$ composed with the mapping $s /\|s\|: \bar{B}_{\eta}^{p} \backslash\{0\} \rightarrow S_{1}^{p-1}$ since their restrictions to this boundary coincide. This glueing may be done in the $C^{\infty}$ category and produces a locally trivial $C^{\infty}$-fibration. We then define $\theta$ to be the result of the glueing of $\mu^{\prime}$ with $s /\|s\| \circ \psi \uparrow: S_{\varepsilon}^{m-1} \cap \psi^{-1}\left(\bar{B}_{\eta}^{p} \backslash\{0\}\right) \rightarrow \bar{B}_{\eta}^{p} \backslash\{0\} \rightarrow S_{1}^{p-1}$, and get that $\left(K_{\varepsilon}, \theta\right)$ is an open book decomposition of $S_{\varepsilon}^{m-1}$.

\section{Remark 2.2.}

In [18, Theorem 1.3] the authors state that if $\operatorname{Sing} \psi \subset V$ and Thom's condition holds along $V$, then there is an empty tube fibration like (7) which blows out to a Milnor fibration on the sphere. As the authors remark themselves [18, pp. 488 and 492], this is a re-formulation of Milnor's results which follows from Milnor's method. There are the following differences with our Theorem 2.1:

6 This observation is also mentioned in [13, Theorem 4.4]. 
(i) The Thom regularity hypothesis of [18, Theorem 1.3] implies our condition $\overline{M(\psi) \backslash V} \cap V=\{0\}$ whereas the converse is presumably not true in general (see below subsection 5.1 for a discussion on this relation and Example 5.1).

(ii) [18, Theorem 1.3] exploits only the fibration outside a tube. We here need a more refined fibration structure inside the tube, which is necessary for having an open book structure as in Definition 1.1.

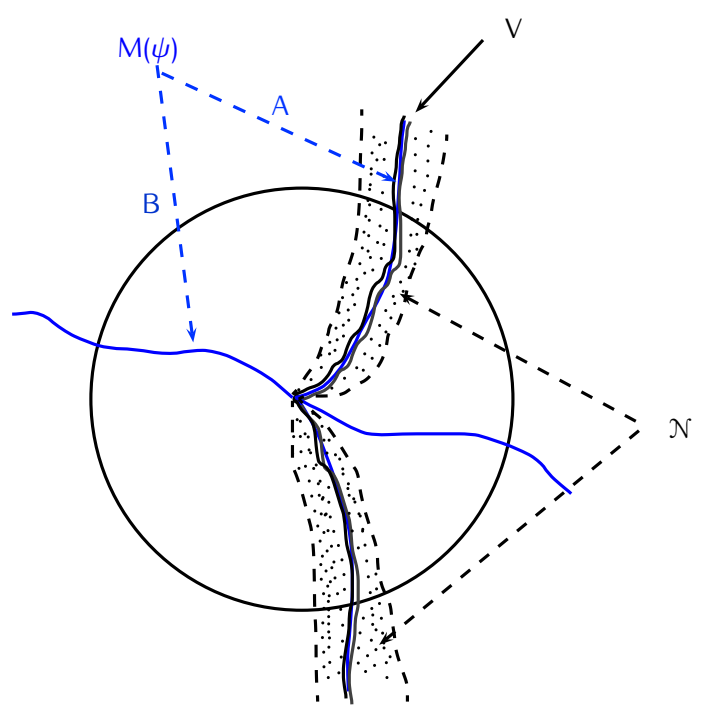

Figure 1.

\section{Proof of Theorem 1.3 and some consequences}

We assume here condition (3) but we do not assume Sing $\psi \subset V$. As explained above in subsection 2.2, the condition $\bar{M}(\psi) \backslash V \cap V=\{0\}$ is equivalent to the existence of a conical neighbourhood $\mathcal{N}$ of $V \backslash\{0\}$ such that $M(\psi) \cap \mathcal{N} \backslash V=\emptyset$. This implies neither the existence of a "full tube" fibration (5), nor the existence of the "empty tube fibration" (7), since our hypothesis (3) allows singularities on the fibres of $\psi$ close to $V$. It is nevertheless enough to imply that the restriction

$$
\psi \uparrow: S_{\varepsilon}^{m-1} \cap \psi^{-1}\left(\bar{B}_{\eta}^{p} \backslash\{0\}\right) \rightarrow \bar{B}_{\eta}^{p} \backslash\{0\}
$$

is a proper submersion, for all small enough $\varepsilon \gg \eta>0$, hence surjective too. On the other hand, the condition that the germ of $M(\psi /\|\psi\|)$ at the origin is empty means that the mapping $\psi /\|\psi\|: S_{\varepsilon}^{m-1} \backslash \psi^{-1}\left(B_{\eta}^{p}\right) \rightarrow S_{1}^{p-1}$ is a proper submersion for any small enough $\varepsilon>0$. Following the proof of Theorem 2.1, we notice that we have here the particularity that the mappings to be glued along the boundary $S_{\varepsilon}^{m-1} \cap \psi^{-1}\left(S_{\eta}^{p-1}\right)$ are induced by the same mapping $\psi /\|\psi\|$ from both sides, hence this glueing is trivial, and of course smooth, with $\theta=\psi /\|\psi\|$. The conclusion follows now just like in the proof of Theorem 2.1.

\section{Example 3.1.}

It was shown in [18] that the mappings of type $f \bar{g}:\left(\mathbb{C}^{2}, 0\right) \rightarrow(\mathbb{C}, 0)$, where $f, g: \mathbb{C}^{2} \rightarrow \mathbb{C}$, are holomorphic functions such that Sing $f \bar{g} \subset V$ has a Milnor fibration $f \bar{g} /|f \bar{g}|: S_{\varepsilon}^{3} \backslash K_{\varepsilon} \rightarrow S^{1}$ and are Thom regular along $V$. Since the Thom regularity implies condition (3) (see also subsection 5.1), it means that the hypotheses of our Theorem 1.3 are satisfied, hence one obtains open book structures with singular binding $\left(K_{\varepsilon}, f \bar{g} /|f \bar{g}|\right)$. 


\subsection{Radial weighted-homogeneous real mappings}

Let us consider the $\mathbb{R}_{+}$-action on $\mathbb{R}^{m}: \rho \cdot x=\left(\rho^{q_{1}} x_{1}, \ldots, \rho^{q_{m}} x_{m}\right)$ for $\rho \in \mathbb{R}_{+}$and $q_{1}, \ldots, q_{m} \in \mathbb{N}^{*}$ relatively prime positive integers. Let $\gamma(x)=\sum_{j=1}^{m} q_{j} x_{j} \partial / \partial x_{j}$ be the corresponding Euler vector field on $\mathbb{R}^{m}$; we have $\gamma(x)=0$ if and only if $x=0$. We say that the mapping $\psi$ is radial weighted-homogeneous of degree $d>0$ if $\psi(\rho \cdot x)=\rho^{d} \psi(x)$ for all $x$ in some neighbourhood of 0 .

\section{Proposition 3.2.}

If $\psi$ is radial weighted-homogeneous and Sing $\psi \subset V$, then $M(\psi /\|\psi\|)=\emptyset$.

Proof. Let us first remark that Sing $\psi \subset V$ implies that $\psi /\|\psi\|: B_{\varepsilon}^{m} \backslash V \rightarrow S_{1}^{p-1}$ is a submersion. We use the following criterion equivalent to $\{0\} \notin M(\psi /\|\psi\|)$ from the proof of [1, Theorem 2.2]: there exists $\varepsilon_{0}>0$ such that rank $\Omega_{\psi}(x)=p$, for all $x \in B_{\varepsilon_{0}}^{m} \backslash V$, where $\Omega_{\psi}(x)$ denotes the $[(p-1) p / 2+1] \times m$ matrix defined as

$$
\Omega_{\psi}(x)=\left[\begin{array}{c}
\omega_{1,2}(x) \\
\vdots \\
\omega_{i, j}(x) \\
\vdots \\
\omega_{p-1, p}(x) \\
x_{1}, \ldots, x_{m}
\end{array}\right]
$$

having in each of the rows the vector $\omega_{i, j}(x)=\psi_{i}(x) \operatorname{grad} \psi_{j}(x)-\psi_{j}(x) \operatorname{grad} \psi_{i}(x)$, for $i, j=1, \ldots, p$ with $i<j$, except for the last row which contains the position vector $\left(x_{1}, \ldots, x_{m}\right)$. Observing that $\left\langle\gamma(x), \operatorname{grad} \psi_{i}(x)\right\rangle=d \cdot \psi_{i}(x)$ for any $i$ and any $x \in B_{\varepsilon_{0}}^{m} \backslash V$, we have

$$
\left\langle\gamma(x), \omega_{i j}(x)\right\rangle=d\left[\psi_{i}(x) \psi_{j}(x)-\psi_{j}(x) \psi_{i}(x)\right]=0,
$$

which means that the Euler vector field $\gamma(x)$ is tangent to the fibres of $\psi /\|\psi\|$. We also have

$$
\langle\gamma(x), x\rangle=\sum_{i} q_{i} x_{i}^{2}>0
$$

for $x \neq 0$. This shows that the position vector $x$ cannot be orthogonal to the tangent space of the fibres of $\psi /\|\psi\|$, which means that the sphere $S_{\varepsilon}^{m-1}$ is transverse to the fibres of $\psi /\|\psi\|$, for any $\varepsilon>0$.

We get the following statement, the proof of which is an immediate consequence of the proof of Theorem 1.3 via Proposition 3.2. It also represents an extension to nonisolated singularities of our previous [1, Theorem 3.1]; see also the proof of [2, Theorem 4.1] and compare with [4, Example 2.1.4].

\section{Corollary 3.3.}

Let $\psi$ be radial weighted-homogeneous with Sing $\psi \subset V$ and satisfying condition (3) with $\operatorname{codim} V=p$. Then $\left(K_{\varepsilon}, \psi /\|\psi\|\right)$ is a (singular) open book decomposition.

\section{Polar weighted-homogeneous mixed functions}

We consider a mixed polynomial ${ }^{7} f(\mathbf{z}, \overline{\mathbf{z}})=\sum_{v, \mu} c_{v, \mu} \mathbf{z}^{v} \overline{\mathbf{z}}^{\mu}$, where $\mathbf{z}=\left(z_{1}, \ldots, z_{n}\right), \overline{\mathbf{z}}=\left(\bar{z}_{1}, \ldots, \bar{z}_{n}\right), \mathbf{z}^{v}=z_{1}^{v_{1}} \cdots z_{n}^{v_{n}}$, $\bar{z}^{\mu}=\bar{z}_{1}^{\mu_{1}} \ldots \bar{z}_{n}^{\mu_{n}}$ and $v=\left(v_{1}, \ldots, v_{n}\right), \mu=\left(\mu_{1}, \ldots, \mu_{n}\right)$ are non-negative integer exponents. As a matter of fact, any mixed polynomial is a real polynomial mapping $\mathbb{R}^{2 n} \rightarrow \mathbb{R}^{2}$, and conversely.

7 The term "mixed polynomial" was introduced by Oka [16]. 
After [3] and [16], we call $f$ polar weighted-homogeneous if there are non-zero integers $p_{1}, \ldots, p_{n}$ and $k$ such that $\operatorname{gcd}\left(p_{1}, \ldots p_{n}\right)=1$ and $\sum_{j=1}^{n} p_{j}\left(v_{j}-\mu_{j}\right)=k$. The corresponding $S^{1}$-action on $\mathbb{C}^{n}$ is

$$
\lambda \cdot(\mathbf{z}, \overline{\mathbf{z}})=\left(\lambda^{p_{1}} z_{1}, \ldots, \lambda^{p_{n}} z_{n}, \lambda^{-p_{1}} \bar{z}_{1}, \ldots, \lambda^{-p_{n}} \bar{z}_{n}\right), \quad \lambda \in S^{1}
$$

Notice that polar weighted-homogeneity and radial weighted-homogeneity are two independent notions.

\subsection{Proof of Theorem 1.4}

We first prove that $\operatorname{Im} f$ contains a small enough disk at $0 \in \mathbb{C}$. Since $f \not \equiv 0$ and $\operatorname{Im} f$ is a semi-algebraic set germ, by the Curve Selection Lemma, the image contains a curve $\gamma$ which intersects the circles $S_{\eta}^{1} \subset \mathbb{C}$ for any small enough radius $\eta>0$. Take now some $a \in S_{\eta}^{1} \cap \gamma$ and $z \in f^{-1}(a)$. Since $f(\lambda \cdot(\mathbf{z}, \bar{z}))=\lambda^{k} f(\mathbf{z}, \bar{z})$, we have $\lambda^{k} a \in \operatorname{Im} f$ for any $\lambda \in S^{1}$. This shows that $\operatorname{lm} f$ contains a disk $D_{\eta_{0}}^{2}$, for some $\eta_{0}>0$ small enough.

The germ at 0 of the set of critical values of $f$ is a semi-algebraic set of dimension $\leq 1$. Take its complement in Im $f$, which is a 2-dimensional semi-algebraic germ at 0 . Applying the above reasoning yields that all values $\neq 0$ are regular, hence their inverse images are manifolds of dimension $2 n-2 \geq 1$. Take now the restriction of $f$ to some small enough sphere $S_{\varepsilon}^{m-1}$. Its image must contain a non-constant curve germ at 0 . Since the $S^{1}$-action preserves the sphere, by the same reasoning as above, the image of $f \uparrow_{S_{\varepsilon}^{m-1}}$ contains a disk $D_{\eta_{0}}^{2}$. The regular values of $f \uparrow_{S_{\varepsilon}^{m-1}}$ are a dense semi-algebraic set and if $a$ is a regular value then $\lambda^{k} a$ is regular too, for any $\lambda \in S^{1}$. Hence all values of the pointed disk $D_{\eta_{0}}^{2} \backslash\{0\}$ are regular. This shows that we have a conical neighbourhood $\mathcal{N}$ of $V$ such that the $\rho$-regularity holds within $\mathcal{N} \backslash V$, so property (3) holds.

In order to prove that $M(f /\|f\|)=\emptyset$ we apply the same reasoning to the mapping $\phi=f /\|f\|$ since the $S^{1}$-action yields $\phi(\lambda \cdot(\mathbf{z}, \bar{z}))=\lambda^{k} \phi(\mathbf{z}, \bar{z})$ and preserves the spheres centred at the origin. Our statement follows now from Theorem 1.3.

\section{Remark 4.1.}

In addition to our hypothesis of polar weighted-homogeneity, if one assumes that $f$ is, moreover, radial weightedhomogeneous and that all weights are positive, then the Milnor fibration induced by $f /|f|$ on the spheres was observed by Oka [16, §5.4] and Cisneros-Molina [3, Proposition 3.4]. These yield property (a) from Section 1 in this particular setting. Note that our Theorem 1.4 drops half of the hypotheses of the preceding results in what concerns property (a), and that our proof has a different flavor. Moreover, it addresses the issue (b) of Section 1 since, according to our definition, the open book structure with singularities in the binding follows only from the conjunction of properties (a) and (b) and not only from one of them. See subsection 5.1 for remarks concerning the conditions which one has to impose near the link, Oka's result [17, Theorem 52] and Example 5.1.

\section{Example 4.2.}

$f(x, y)=x^{4} \bar{y}^{2}+y^{2}$ is not radial weighted homogeneous but a polar homogeneous mixed function germ $\left(\mathbb{C}^{2}, 0\right) \rightarrow(\mathbb{C}, 0)$, and we have Sing $f=V=\{y=0\}$. According to Theorem 1.4, $f /|f|$ defines on $S^{3}$ an open book structure with singular binding.

\section{More examples with nonisolated singularities}

\subsection{Condition (3) and Thom regularity condition}

It is well known that if $V=\psi^{-1}(0)$ may be endowed with a stratification $\mathcal{S}$ such that in a sufficiently small ball $B_{\epsilon}$ the pair $\left(B_{\epsilon} \backslash V, S\right)$ satisfies Thom's condition $\left(\mathrm{a}_{\psi}\right)$, for all $S \in \mathcal{S}$, then the stratified transversality of $V$ to all small enough spheres implies the transversality of the spheres to the nearby fibres in some neighbourhood $\mathcal{N}$ of $V \backslash\{0\}$. This transversality is equivalent to condition (3) and by the proof of Theorem 1.3 we conclude the existence of the full tube fibration on all small enough spheres (9). This well-known observation may be traced back at least to [7]; see also [8, 11]. 
It is conjecturally possible that the condition (3) does not imply the Thom $\left(\mathrm{a}_{\psi}\right)$-regularity, but we could not find an example yet. Let us at least point out two situations where one proves directly the weaker condition (3). One of them is contained in the proof of [17, Lemma 51] in the setting of mixed functions $f$, where Oka shows the existence of a full tube fibration for a special class of mappings, namely the super strongly non-degenerate mixed functions. This is a condition which allows Oka to prove in [17, Theorem 52] the existence of the Milnor fibration on spheres (by extending Milnor's method) and its equivalence to the tube fibration. Altogether these properties yield, in our terminology, an open book structure with singular binding induced by the mapping $f /|f|$.

Another example of computation is the following one, where the mapping is not a mixed function, hence it is different from all the previously mentioned situations of [17, Theorem 52] or of Theorem 1.4.

\section{Example 5.1.}

Let $f: \mathbb{R}^{3} \rightarrow \mathbb{R}^{2}, f(x, y, z)=\left(y^{4}-z^{2} x^{2}-x^{4}, x y\right)$. Then $V(f)$ is the real line $\left\{(x, y, z) \in \mathbb{R}^{3}: x=y=0\right\}$ and Sing $f=V(f)$. Let us show (3). If this were not true, then, by the Curve Selection Lemma, there are some analytic curves $x(t), y(t), z(t), a(t), b(t)$ defined on a small enough interval $] 0, \varepsilon\left[\right.$ such that $\lim _{t \rightarrow 0} x(t)=\lim _{t \rightarrow 0} y(t)=0, \lim _{t \rightarrow 0} z(t)=z_{0} \neq 0$ and

$$
x(t)=a(t)\left(-4 x^{3}(t)-2 x(t) z^{2}(t)\right)+b(t) y(t), \quad y(t)=4 a(t) y^{3}(t)+b(t) x(t), \quad z(t)=-2 a(t) z(t) x^{2}(t)
$$

Let $x(t)=x_{0} t^{\beta}+$ h.o.t., where $x_{0} \neq 0$ and $\beta \in \mathbb{N}$. From the third equality and from $z(t) \rightarrow z_{0}$ we get $a(t)=$ $-t^{-2 \beta} /\left(2 x_{0}^{2}\right)+$ h.o.t. We eliminate $b(t)$ from the first two lines and get

$$
y^{2}(t)-x^{2}(t)=a(t)\left(4 y^{4}(t)+4 x^{4}(t)+2 x^{2}(t) z^{2}(t)\right)
$$

From this equality, since $2 x^{2}(t) z^{2}(t) a(t) \rightarrow-z_{0}^{2}<0$ and $\operatorname{ord}_{t}\left(a(t) x^{4}(t)\right)=2 \beta>0$, and $y^{2}(t)-x^{2}(t) \rightarrow 0$, we get $y^{4}(t)=-x_{0}^{2} z_{0}^{2} t^{2 \beta} / 2+$ h.o.t., which yields a sign contradiction.

\subsection{A real Thom-Sebastiani type statement}

In order to build subsequent examples, we show the following.

\section{Proposition 5.2.}

Consider two mappings in separate variables, $\psi:\left(\mathbb{R}^{m}, 0\right) \rightarrow\left(\mathbb{R}^{p}, 0\right)$ and $\phi:\left(\mathbb{R}^{n}, 0\right) \rightarrow\left(\mathbb{R}^{p}, 0\right)$, such that Sing $\psi \subset V(\psi)$ and Sing $\phi \subset V(\phi)$, and both $V(\psi)$ and $V(\phi)$ have codimension $p$. Assume that $\psi$ and $\phi$ satisfy the Thom regularity condition at $V(\psi)$ and $V(\phi)$, respectively. Then $(\psi+\phi):\left(\mathbb{R}^{m} \times \mathbb{R}^{n}, 0\right) \rightarrow\left(\mathbb{R}^{p}, 0\right)$ satisfies the Thom regularity condition and there exists a higher open book structure with singular binding $\left(K_{\psi+\phi}, \theta\right)$ on $S_{\varepsilon}^{m+p-1}$, which is independent of $\varepsilon>0$ small enough, up to isotopies. If moreover $\psi$ and $\phi$ are radial weighted-homogeneous then $\left(K_{\psi+\phi},(\psi+\phi) /\|\psi+\phi\|\right)$ is a higher open book with singular binding.

Proof. From Sing $\psi \subset V(\psi)$ and Sing $\phi \subset V(\phi)$ it follows, by checking the rank of the Jacobian matrix, that Sing $(\psi+\phi) \subset$ Sing $\psi \times$ Sing $\phi \subset V(\psi) \times V(\phi) \subset V(\psi+\phi) \subset \mathbb{R}^{m} \times \mathbb{R}^{n}$. The sum of separate variables mappings which both satisfy the Thom regularity condition has the same property. Indeed, if $\mathcal{W}_{1}$ and $\mathcal{W}_{2}$ denote some Thom stratifications of $V(\psi)$ and $V(\phi)$ respectively, then the product stratification $\mathcal{W}_{1} \times \mathcal{W}_{2}$ satisfies the Thom $\left(a_{\psi+\phi}\right)$-regularity condition. To prove this, starting with the definition we consider the limits of tangent spaces to the fibres of $\psi+\phi$ along a sequence of points $\left(x_{i}, y_{i}\right) \in \mathbb{R}^{m} \times \mathbb{R}^{n}$ tending to some point $(\alpha, \beta) \in W_{1} \times W_{2}$ for some strata $W_{1} \in \mathcal{W}_{1}$ and $W_{2} \in \mathcal{W}_{2}$, at least one of which is of positive dimension. We work with sequences such that $\psi\left(x_{i}\right)+\phi\left(y_{i}\right) \neq 0$ for any $i>0$.

If $\psi\left(x_{i}\right) \neq 0$ and $\phi\left(y_{i}\right) \neq 0$ for any high enough index $i$, then we have the inclusion $T_{\left(x_{i}, y_{i}\right)}(\psi+\phi) \supset T_{x_{i}} \psi \times T_{y_{i}} \phi$. Else, if say $\psi\left(x_{i}\right)=0$ for all $i$ large enough, then $\phi\left(y_{i}\right) \neq 0$ for those indices $i$, and we have the inclusion $T_{\left(x_{i}, y_{i}\right)}(\psi+\phi) \supset T_{x_{i}} W_{x_{i}} \times T_{y_{i}} \phi$, where $W_{x_{i}}$ denotes the stratum of $V(\psi)$ such that $x_{i} \in W_{x_{i}}$. Proving this inclusion essentially amounts to checking that for any $v \in T_{x_{i}} W_{x_{i}}$ one has $D \psi\left(x_{i}\right) v=0$. Indeed, if one considers a path $\alpha(t)$ within $W_{x_{i}}$ with $\partial \alpha /\left.\partial t\right|_{t=0}=v$ then we have $\psi \circ \alpha \equiv 0$. Thus

$$
\left.\frac{\partial(\psi \circ \alpha)}{\partial t}\right|_{t=0}=0
$$


and the latter is also equal to $D \psi\left(x_{i}\right) v$.

In both situations, taking limits we get the desired inclusion $\lim _{i \rightarrow \infty} T_{\left(x_{i}, y_{i}\right)}(\psi+\phi) \supset W_{1} \times W_{2}$, due to the Thom regularity of $\psi$ and $\phi$. Finally, one may refine the product stratification $\mathcal{W}_{1} \times \mathcal{W}_{2}$ to a Whitney (a)-regular stratification so that the singular locus Sing $(\psi+\phi)$ is a union of strata. This is possible by the classical theory of Whitney stratifications, see e.g. [6] for the algorithm. By construction, this refinement is a Thom regular stratification of $V(\psi+\phi)$. This finishes the proof of our claim. Taking now into account also the codimension $p$ condition of the statement (not used up to now), it appears that the map $\psi+\phi$ verifies the hypotheses of Theorem 2.1 in view of our above remarks in subsection 5.1 about the Thom condition. The first claim of our statement follows.

Furthermore, if each mapping is radial weighted-homogeneous, the separate variable sum $\psi+\phi$ has the same property (of course, the weights have to be multiplied by some integers if the weighted degrees of $\psi$ and $\phi$ are not the same). Thus one may apply Corollary 3.3 and get the second claim of the statement.

\section{Example 5.3.}

Let $h: \mathbb{R}^{3} \times \mathbb{C}^{n} \rightarrow \mathbb{R}^{2}, h=f(x, y, z)+g\left(w_{1}, \ldots, w_{n}\right)$, where $f: \mathbb{R}^{3} \rightarrow \mathbb{R}^{2}$ is from Example 5.1 and $g: \mathbb{C}^{n} \rightarrow \mathbb{C}=\mathbb{R}^{2}$ is a sum of monomials $\sum_{i=1}^{n} a_{i} m_{i}$, where $m_{i}=w_{i}^{k_{i}}$ or $m_{i}=\bar{w}_{i}^{k_{i}}$ with complex coefficients $a_{i}$. Then $g$ is radial weightedhomogeneous and has Thom property by Proposition 5.2, or by the fact that this map has isolated singularity. As for $f$, it is not radial weighted-homogeneous, we have seen above that it satisfies the condition $\overline{M(f) \backslash V} \cap V=\{0\}$ but we need the Thom regularity condition in order to apply Proposition 5.2 (first claim). We claim that $f$ also satisfies the Thom $\left(\mathrm{a}_{f}\right)$-regularity condition at Sing $f \backslash\{0\}$. Let us fix some point $\left(0,0, z_{0}\right) \in \operatorname{Sing} f=V(f), z_{0} \neq 0$, and choose an analytic

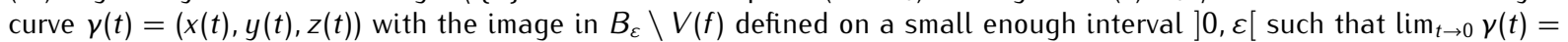
$\left(0,0, z_{0}\right)$. For any $t$, the normal vector field $v_{1}(t)=(y(t), x(t), 0)$ to the fibres of $f$ is orthogonal to the direction $(0,0,1)$ of the line $V(f)$. Let us consider the normal vector field to the fibres of $f, v_{2}(t)=\left(-4 x^{3}(t)-2 x(t) z^{2}(t), 4 y^{3}(t),-2 z(t) x^{2}(t)\right)$, which is independent of $v_{1}(t)$. What we actually need to show is that the limit of the vector product

$$
\frac{v_{1}(t)}{\left\|v_{1}(t)\right\|} \wedge \frac{v_{2}(t)}{\left\|v_{2}(t)\right\|}
$$

is a nonzero vector in the space spanned by $(0,0,1)$. One has

$$
v(t)=v_{1}(t) \wedge v_{2}(t)=\left(-2 x^{3}(t) z(t), 2 z(t) x^{2}(t) y(t), 4 y^{4}(t)+2 x^{2}(t) z^{2}(t)+4 x^{4}(t)\right) .
$$

We have the following two situations:

(i) If $\operatorname{ord}_{t} x(t) \leq 2 \operatorname{ord}_{t} y(t)$ or if $y(t) \equiv 0$, we have

$$
\lim _{t \rightarrow 0} \frac{4 y^{4}(t)+2 x^{2}(t) z^{2}(t)+4 x^{4}(t)}{x^{2}(t)}=2 z_{0}^{2}+\lim _{t \rightarrow 0} \frac{4 y^{4}(t)}{x^{2}(t)}>0
$$

$$
\text { and } \lim _{t \rightarrow 0}(-2 x(t) z(t), 2 z(t) y(t))=(0,0) .
$$

(ii) If $\operatorname{ord}_{t} x(t)>2 \operatorname{ord}_{t} y(t)$ or if $x(t) \equiv 0$, we have

$$
\lim _{t \rightarrow 0} \frac{4 y^{4}(t)+2 x^{2}(t) z^{2}(t)+4 x^{4}(t)}{y^{4}(t)}=4 \neq 0, \quad \lim _{t \rightarrow 0} \frac{\left(-2 x^{3}(t) z(t), 2 z(t) x^{2}(t) y(t)\right)}{y^{2}(t)}=(0,0)
$$

These show that the tangent space to the line $\operatorname{Sing} f$ is contained in the limit of the tangent spaces to the fibres of $f$, hence that $f$ satisfies the Thom $\left(a_{f}\right)$-regularity condition at $V(f) \backslash\{0\}$. 


\subsection{Added in proof}

We have seen above that the Thom regularity condition along $V$ is not necessary for having the Milnor fibration in a conical neighbourhood of $V$, as explained in the first lines of Section 3. Indeed, consider for instance the mixed function $f: \mathbb{C}^{2} \rightarrow \mathbb{C}, f(x, y)=x y \bar{x}$. It is polar homogeneous, hence verifies condition (3) and our Theorem 1.4, but one can easily check that there is no Thom $\left(\mathrm{a}_{f}\right)$ stratification of $V$. A deformation of this example in three variables has been suggested by A. Parusiński during a recent workshop in Oberwolfach [26], namely $g(x, y, z)=\left(x+z^{3}\right) y \bar{x}$. This is polar weighted-homogeneous and verifies condition (3) and our Theorem 1.4, but again there is no Thom $\left(a_{f}\right)$ stratification of $V$. These provide a positive answer to our conjecture formulated above in the second paragraph of Section 5, i.e. that Thom's regularity condition is strictly stronger than condition (3). The latter also provides a simple counter-example to a statement conjectured by Pichon and Seade in [18, p. 494] and proved as the main result in a subsequent paper of the same authors.

\section{Acknowledgements}

Raimundo N. Araújo dos Santos acknowledges support from the Brazilian CNPq, PQ-2, proc. num. 305183/2009-5, and FAPESP proc. num. 2009/14383-3. Mihai Tibăr acknowledges support from the French Agence Nationale de la Recherche, grant ANR-08-JCJC-0118-01.

\section{References}

[1] Araújo dos Santos R., Tibăr M., Real map germs and higher open book structures, Geom. Dedicata, 2010, 147, 177-185

[2] Araújo dos Santos R., Tibăr M., Real map germs and higher open books, preprint available http://arxiv.org/abs/ 0801.3328

[3] Cisneros-Molina J.L., Join theorem for polar weighted homogeneous singularities, In: Singularities II, Cuernavaca, January 8-26, 2007, Contemp. Math., 475, American Mathematical Society, Providence, 2008, 43-59

[4] Cisneros-Molina J.L., Seade J., Snoussi J., Milnor fibrations and $d$-regularity for real analytic singularities, Internat. J. Math., 2010, 21(4), 419-434

[5] Ehresmann C., Les connexions infinitésimales dans un espace fibré différentiable, In: Colloque de Topologie (Espaces Fibrés), Bruxelles, 1950, Georges Thone, Liège; Masson et Cie., Paris, 1951, 29-55

[6] Gibson C.G., Wirthmüller C., du Plessis A.A., Looijenga E.J.N., Topological Stability of Smooth Mappings, Lecture Notes in Math., 552, Springer, Berlin-New York, 1976

[7] Hamm H.A., Lê D.T., Un théorème de Zariski du type de Lefschetz, Ann. Sci. École Norm. Sup., 1973, 6(3), 317-355

[8] Hironaka H., Stratification and flatness, In: Real and Complex Singularities, Oslo, August 5-25, 1976, Sijthoff and Noordhoff, Alphen aan den Rijn, 1977, 199-265

[9] Jacquemard A., Fibrations de Milnor pour des applications réelles, C. R. Acad. Sci. Paris Sér. I Math., 1983, 296(10), 443-446

[10] Jacquemard A., Fibrations de Milnor pour des applications réelles, Boll. Un. Mat. Ital. B, 1989, 3(3), 591-600

[11] Lê D.T., Some remarks on relative monodromy, In: Real and Complex Singularities, Oslo, August 5-25, 1976, Sijthoff and Noordhoff, Alphen aan den Rijn, 1977, 397-403

[12] Looijenga E., A note on polynomial isolated singularities, Indag. Math., 1971, 33, 418-421

[13] Massey D.B., Real analytic Milnor fibrations and a strong Łojasiewicz inequality, In: Real and Complex Singularities, London Math. Soc. Lecture Note Ser., 380, Cambridge University Press, Cambridge, 2010, 268-292

[14] Milnor J., Singular Points of Complex Hypersurfaces, Ann. of Math. Studies, 61, Princeton University Press, Princeton, 1968

[15] Némethi A., Zaharia A., Milnor fibration at infinity, Indag. Math., 1992, 3(3), 323-335

[16] Oka M., Topology of polar weighted homogeneous hypersurfaces, Kodai Math. J., 2008, 31(2), 163-182 
[17] Oka M., Non-degenerate mixed functions, Kodai Math. J., 2010, 33(1), 1-62

[18] Pichon A., Seade J., Fibred multilinks and singularities $f \bar{g}$, Math. Ann., 2008, 342(3), 487-514

[19] Ruas M.A.S., Araújo dos Santos R.N., Real Milnor fibrations and (c)-regularity, Manuscripta Math., 2005, 117(2), 207-218

[20] Ruas M.A.S., Seade J., Verjovsky A., On real singularities with a Milnor fibration, In: Trends in Singularities, Trends Math., Birkhäuser, Basel, 2002, 191-213

[21] Tibăr M., On the monodromy fibration of polynomial functions with singularities at infinity, C. R. Acad. Sci. Paris Sér. I Math., 1997, 324(9), 1031-1035

[22] Tibăr M., Regularity at infinity of real and complex polynomial functions, In: Singularity Theory, Liverpool, August 18-24, 1996, London Math. Soc. Lecture Note Ser., 263, Cambridge University Press, Cambridge, 1999, 249-264

[23] Tibăr M., Polynomials and Vanishing Cycles, Cambridge Tracts in Math., 170, Cambridge University Press, Cambridge, 2007

[24] Winkelnkemper H.E., Manifolds as open books, Bull. Amer. Math. Soc., 1973, 79(1), 45-51

[25] Wolf J.A., Differentiable fibre spaces and mappings compatible with Riemannian metrics, Michigan Math. J., 1964, 11(1), 65-70

[26] Mini-Workshop: Topology of Real Singularities and Motivic Aspects, Oberwolfach, September 30-October 3, 2012, Oberwolfach Report No. 48/2012, Mathematisches Forschungsinstitut Oberwolfach, 2012, DOI: $10.4171 / O W R / 2012 / 48$ 